\title{
Detection of minority variants within bovine respiratory syncytial virus populations using oligonucleotide-based microarrays
}

\author{
Véronique Leberre ${ }^{\mathrm{a}, \mathrm{b}, *}$, Eric Baranowski ${ }^{\mathrm{c}}$, Martine Deplanche ${ }^{\mathrm{c}}$, \\ Lidwine Trouilh ${ }^{\mathrm{b}}$, Jean Marie François ${ }^{\mathrm{a}, \mathrm{b}}$

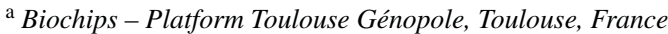 \\ b UMR 5504 and UMR 792 d'Ingénierie des Systèmes, Biologiques et Procédés, CNRS-INRA-INSA, Toulouse, France \\ ${ }^{\mathrm{c}}$ UMR1225 Interactions Hôtes-Agents Pathogènes, INRA-ENVT, Ecole Nationale Vétérinaire de Toulouse, Toulouse, France
}

\footnotetext{
* Corresponding author at: UMR 5504 and UMR 792 d'Ingénierie des Systèmes, Biologiques et Procédés, CNRS-INRA-INSA, Toulouse, France.

Tel.: +335615596 87; fax: +33561559400.

E-mail address: leberre@insa-toulouse.fr (V. Leberre).
}

\begin{abstract}
Microarray technology, originally developed for highly parallel examination of gene expression is regarded as a potential tool in prognosis and diagnosis. With respect to a discrimination analysis, difference as small as one nucleotide base can be distinguished using oligonucleotide-based microarrays. However, this degree of specificity is dependent on several parameters, including the size of the oligoprobes and the sequence context of the probes (e.g. local melting temperature), hybridization conditions and to some extent the chemistry of the glass slides onto which the probes are deposited. Using bovine respiratory syncytial virus (BRSV) as a model study, an oligonucleotide-based microarray approach was developed to measure the relative abundance of a particular single nucleotide variant within mixed BRSV populations. Using this technology, we show that it is possible to discriminate at a rate of $1 \%$, minority variants in a BRSV population.
\end{abstract}

Keywords: Oligo-microarrays; BRSV; Discrimination

\section{Introduction}

Human and bovine respiratory syncytial viruses (HRSV and BRSV) are closely related paramyxoviruses responsible for severe lower respiratory tract disease in infants and calves, respectively. The bronchiolitis and pneumonia caused by BRSV in calves are similar clinically, pathologically, and epidemiologically to the disease caused by HRSV in children.

Because of the high mutation rate operating during RNA genome replication, BRSV populations are genetically heterogeneous (Deplanche et al., 2007) and exhibit an important potential for variation which is reflected in its antigenic diversity. Despite evidence of the highly heterogeneous nature of RNA viral populations, the consensus sequence is often the unique criteria used to characterize these genome populations. Work with different viral systems has highlighted the significance of the mutant spectrum - the distribution of variants continuously generated during the error-prone replication of RNA genomes for the study of RNA viral quasispecies and their adaptability to changing environments (Domingo et al., 2006). Analysis of the mutant spectrum can also provide valuable information on virus response to antiviral treatments (Domingo and Gomez, 2007), and may find applications for the quality control of attenuated vaccines, including the risk associated with virulence reversion (Amexis et al., 2001). This calls for methods capable of determining distributions of mutations within RNA virus genome populations that should be rapid and cost-effective, but preferentially high throughput. Sequencing of a viral genomic region from a collection of biological clones (virus from individual plaques formed on cell monolayers) and/or molecular clones (RT-PCR products cloned in E. coli) is a common method to generate coarse-grained pictures of the mutant spectrum. Alternatively, Q-PCR assays have been developed to determine the frequency of particular mutant genomes within viral populations. Recently, a number of Q-PCR-derived methods have been developed for the assessment of genetic stability and quantitative monitoring of genetic changes in RNA viruses of clinical interest 
(Wood and Macadam, 1997; Amexis et al., 2001). The MAPREC method, which combines Q-PCR to enzyme restriction cleavage, has proven to be a sensitive method (Chumakov et al., 1992), yet is restricted to a limited number of known mutations (Wood and Macadam, 1997). Amexis et al. (2001) proposed to combine MAPREC with matrix-assisted laser desorption/ionization time-of-flight (MALDI-TOF) mass spectrometry (DNA MassArray). However, major disadvantages of these methods are that they are comparatively labour intensive, low throughput and expensive.

We have recently shown that single nucleotide changes can be distinguished using oligonucleotide-based microarrays (Le Berre et al., 2003), suggesting that monitoring of genetic heterogeneity within viral quasispecies could be achieved using DNA microarrays. This technique permits simultaneous interrogation with a large number of probe sequences with high robustness and at a lower price per assay. The degree of specificity can be influenced by different parameters, including the size of the oligoprobes or their sequence context (e.g. local melting temperature), hybridization conditions and to some extent the chemistry of the glass slides onto which the probes are deposited. The literature is relatively vague with respect to these technical questions, as for instance probe sizes from 13 up to 50 mers have been used to diagnose various viral species (Warsen et al., 2004; Ryabinin et al., 2006; Martin et al., 2006; Palacios et al., 2007).

Using BRSV as a model study, we investigated optimal conditions to discriminate between parental and mutants of BSRV at a specific genome position using DNA oligo-microarrays. Using this technology, we show that it is possible to discriminate at a rate of $1 \%$, single nucleotide minority variants in a BRSV population.

\section{Materials and methods}

\subsection{Virus populations}

BRSV populations W2BT10 and W2HEp58 derived from strain W2-00131 (Deplanche et al., 2007). Virus population W2BT10 was obtained upon propagation of BRSV W2-00131 in bovine turbinate (BT; ATCC reference CRL1390) cells for 10 passages. BRSV W2HEp58 was derived from population W2BT10 following virus amplification in baby hamster kidney 21 (BHK-21; ATCC reference CCL-10) cells and long-term propagation (58 passages) in human epithelial 2 (HEp-2; ATCC reference CCL-23) cells.

\subsection{Oligonucleotides design}

Two sets of oligonucleotides specific to BRSV genomic region encoding phosphoprotein $\mathrm{P}$ (GeneBank accession no. AF092942) were designed (Table 1). The probe lengths of the first set was 14-15 mers and a second set from 19-23. Unless otherwise stated, these probes were flanked with a 15 mer poly $\mathrm{dT}$ at their $5^{\prime}$-extremity. The single nucleotide that discriminates between BRSV parental (W2BT10) and the BRSV mutant (W2HEp58) genomes (position 2686 on the RNA genome) was always positioned at the centre of the 14-22 mers oligonu-
Table 1

Oligonucleotides specific to BRSV genome

\begin{tabular}{|c|c|c|}
\hline Oligonucleotide & Sequence $\left(5^{\prime} \rightarrow 3^{\prime}\right)^{\mathrm{a}}$ & $\begin{array}{l}\text { Genomic } \\
\text { position }^{\text {b }}\end{array}$ \\
\hline P112-1 & TTCTTCATTATTGT & $2692-2679$ \\
\hline P112-2 & TTCTTCECTTATTGT & $2692-2679$ \\
\hline P112-3 & CAATAATGAAGAAG & $2680-2693$ \\
\hline P112-4 & CAATAAGGAAGAAG & $2680-2693$ \\
\hline P112-5 & ACAATAATGAAGAAG & $2679-2693$ \\
\hline P112-6 & ACAATAAGGAAGAAG & $2679-2693$ \\
\hline P112-7 & TTCTTCATTATTGTC & $2692-2678$ \\
\hline P112-8 & TTCTTCÉTTATTGTC & $2692-2678$ \\
\hline P112-9 & CAATAAT̄GAAGAAGA & $2680-2694$ \\
\hline P112-10 & CAATAAGGAAGAAGA & $2680-2694$ \\
\hline P112-19 wt & TGACAATAATGAAGAAGAA & $2677-2695$ \\
\hline P112-19 mut & TGACAATAAGGAAGAAGAA & $2677-2695$ \\
\hline P112-21 wt & TTGACAATAATGAAGAAGAAT & $2676-2696$ \\
\hline P112-21 mut & TTGACAATAAGGAAGAAGAAT & $2676-2696$ \\
\hline P112-23 wt & TTTGACAATAATGAAGAAGAATC & $2675-2697$ \\
\hline P112-23 mut & TTTGACAATAA $\underline{\text { GGAAGAAGAATC }}$ & $2675-2697$ \\
\hline F70-23 & AATGTGTGTAAĀAGTACTGATTC & $5768-5790$ \\
\hline F218-19 & CCAACATAGTAACTGTGAT & $6213-6231$ \\
\hline
\end{tabular}

${ }^{a}$ The mutated position is underlined.

b The numbering corresponds to the reference sequence of BRSV genome (GeneBank accession no. AF092942).

cleotides (not taking into account the flanking spacer). Two additional BRSV-specific oligonucleotides (F70-23 and F21819) mapping outside of phosphoprotein $P$ coding region were used as a control. The oligonucleotides were purchased from Operon.

\subsection{Preparation of target DNA}

Target DNA was obtained by PCR amplification from plasmid DNA or cDNA templates using labelled oligonucleotides $5^{\prime}$ Cy5-GGAAACTATAGAAACATTTGACAATAA-3' (BRSV genome position 2659-2685) and 5'-Cy5-ATATGAGTAGCTTGATTCTTCTTC-3' (BRSV genome position 2710-2687). cDNA templates were produced by retrotranscription (RT) of BRSV genomic RNA using oligonucleotide N2.1 as described by Valarcher et al. (1999). To construct plasmid DNA templates encoding BRSV sequences derived from W2BT10 (parental) and W2HEp58 (mutant) populations, BRSV genomic region spanning nucleotides 1959-3333 was amplified by RT-PCR using oligonucleotides 5'-TGGTCATGCCAGCGTACAAGC$3^{\prime}$ and $5^{\prime}$-CATAGGAACCCATATTGTRAGAGATGC-3' and cloned into pCRII-TOPO vector (Invitrogen). Plasmid DNA construction was verified by nucleotide sequencing of the PCRamplified BRSV fragments. Heterogeneous target DNA samples were produced by PCR amplification using different ratios of parental and mutant BRSV cDNAs as DNA templates (Table 2). A SYBR green-based Q-PCR assay was used to standardize BRSV cDNA templates derived from populations W2BT10 (parental) and W2HEp58 (mutant). Oligonucleotides $5^{\prime}$-ATTCGTACATTTTGGCATTGCTC-3' and 5'-TTCCTTCWACCCTACTACCTCCTCT-3' were designed to amplify a $59 \mathrm{bp}$ product from BRSV cDNA (BRSV genome position 1809-1867) using Primer Express v2.0 software (Applied 
Table 2

Parental and mutant BRSV cDNAs ratios in heterogeneous target DNA samples

\begin{tabular}{lc}
\hline Heterogeneous sample & cDNAs ratio $^{\mathrm{a}}$ \\
\hline 100 & $100: 0$ \\
99 & $99: 1$ \\
90 & $93: 7$ \\
50 & $60: 40$ \\
10 & $15: 85$ \\
1 & $1: 99$ \\
0 & $0: 100$
\end{tabular}

${ }^{\mathrm{a}}$ [BRSV WT cDNA]/[BRSV mutant cDNA].

Biosystems). The reaction was carried out using the qPCR ROX-\&GO Green reaction mix (QBIOgene). Thermal cycling was performed with the GeneAmp 5700 (Applied Biosystems) under the following conditions: $95^{\circ} \mathrm{C}$ for $15 \mathrm{~min}$ followed by 40 cycles of $94^{\circ} \mathrm{C}$ for $15 \mathrm{~s}$ and $59^{\circ} \mathrm{C}$ for $30 \mathrm{~s}$. Standard curves were generated from 10 -fold dilutions of plasmid DNA template encoding parental BRSV sequence.

\subsection{Microarray printing}

Lyophylised oligonucleotides were dissolved in the Nexterion ${ }^{\mathrm{TM}}$ Spot solution from Schott- $0.005 \% \operatorname{SDS}(20 \mu \mathrm{M})$. They were printed on epoxy-silane-derivatized glass slides
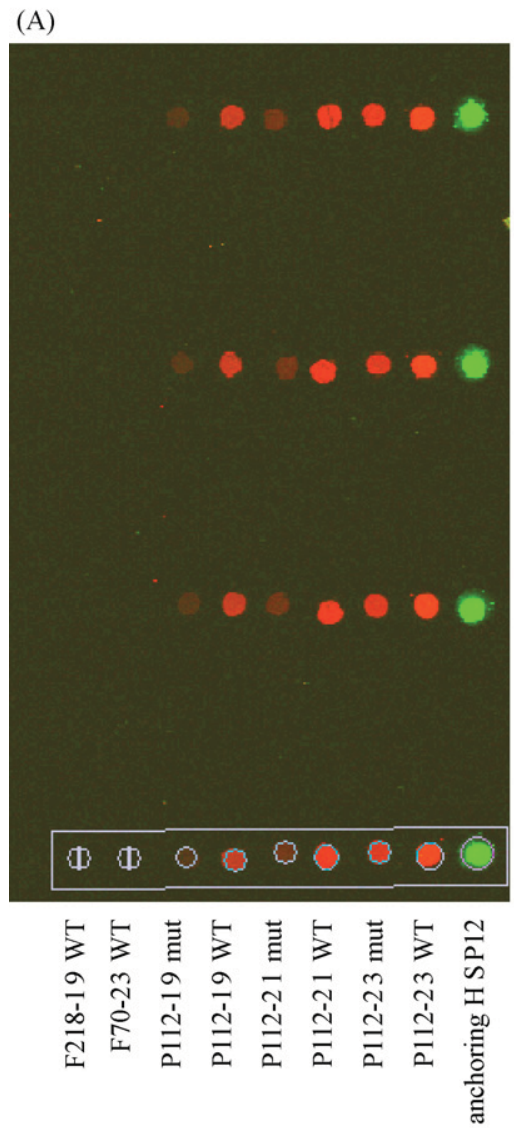
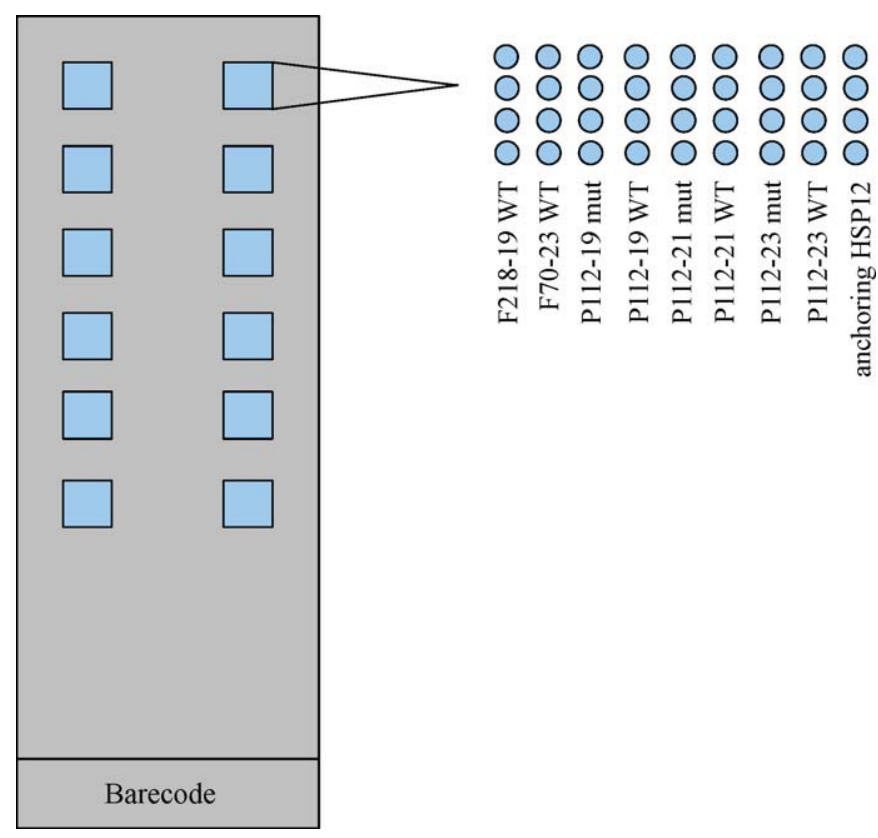

Fig. 1. Scheme of probes spotting on epoxy-activated glass slides. Oligonucleotides were spotted in quadruplicate, with a centre to centre distance of $250 \mu \mathrm{m}$. A Cy3-labelled yeast HSP12 oligonucleotide (5'-Cy3-GACACGACCGGAAACATATT- $3^{\prime}$ ) was spotted in quadruplicate at the right side of square to allow anchoring of the feature for image capture by the GenePix6.0 software. This pattern was repeated 12 times to fit with the 16 well-superstructure seal strips from Schott Nexterion used in the hybridization step.

(B)

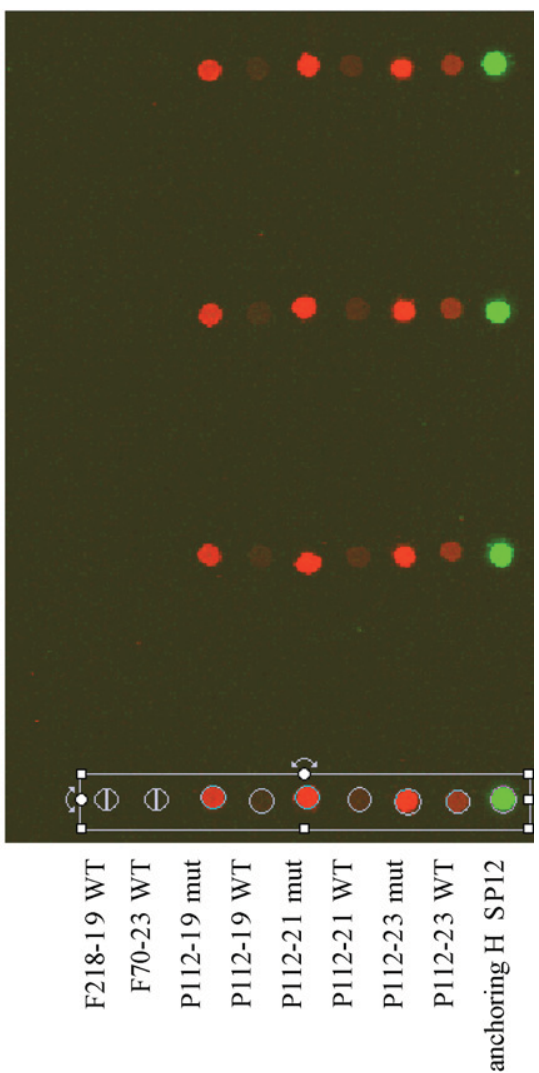

Fig. 2. Hybridization patterns of BRSV parental (A) and mutant (B) target DNA on the oligonucleotides arrays. The Cy5-labelled targets were produced by RT-PCR on a BRSV genomic region encompassing position 2686. These amplicons were hybridized at $37^{\circ} \mathrm{C}$ for $4 \mathrm{~h}$. Images were captured by the laser scanner Genepix $4000 \mathrm{~A}$ at a PMT of 600 at $635 \mathrm{~nm}$ and 450 at $532 \mathrm{~nm}$. 
(Nexterion ${ }^{\circledR}$ HiSens E from Schott) by mechanical spotting with a robot (Q-Array mini from Genetix). Further technical information relating to this robot can be found at P.O. Brown's website (http://cmgm.stanford.edu/pbrown/mguide/index.html). The print head on the arrayer uses 'quill' pins SMP3 from Arraylt, whose contact on the surface delivers about $1 \mathrm{nl}$ of DNA solution per spot, with a size diameter of about $100 \mu \mathrm{m}$ under this condition. Oligoprobes were spotted in quadruplicate, with a centre to centre distance of $250 \mu \mathrm{m}$. An anchoring Cy3-labelled oligonucleotide derived from yeast HSP12 gene (5'-Cy3-GACACGACCGGAAACATATT-3') was spotted in quadruplicate, on the right side of the probes according to Fig. 1. Twelve replicates of this grid were spotted on the slide. The oligo-microarrays were kept in a plastic container and stored in a desiccator at room temperature until use.

\subsection{Hybridization and scanning}

Pre-hybridization of the printing area was carried out by immersing array in Pronto ${ }^{\mathrm{TM}}$ Universal Pre-hybridization Solution (Corning) for $30 \mathrm{~min}$ at $37^{\circ} \mathrm{C}$. The slides were then transferred to a fresh container and wash for 2 min with a $0.2 \times$ SSC solution, then transferred to another container and wash for 2 min with $0.1 \times \mathrm{SSC}$, then dried by centrifugation. Hybridization was carried out at $37^{\circ} \mathrm{C}$ or $55^{\circ} \mathrm{C}$ for $2 \mathrm{~h}$ or $4 \mathrm{~h}$ in the Eppendorf $^{\circledR}$ ThermoStat Plus equipped with exchangeable thermoblock for four slides. A silicon joint was stick on the slide to delimit the 12 zones of hybridization (Fig. 1). A $3 \mu$ l solution of PCR target was mixed with $40 \mu$ l of Pronto ${ }^{\text {TM }}$ short oligohybridization solution (Corning) and with $3 \mu$ l of sonicated DNA salmon sperm. After denaturation at $95^{\circ} \mathrm{C}$ for $5 \mathrm{~min}$, the mix was transferred onto the delimited zone of the printed slide. Post-hybridization washes were carried out by incubating the slides under agitation, $5 \mathrm{~min}$ in a $2 \times \mathrm{SSC} / 0.1 \% \mathrm{SDS}$ solution, followed by $2 \mathrm{~min}$ in a $0.2 \times \mathrm{SSC}$ solution, then $2 \mathrm{~min}$ into $0.1 \times$ SSC solution. The slide was then dried by centrifugation. Fluorescence signals were detected by a laser scanner (GenePix 4000A from Axon Instrument) and analysed with the GenePix 6.01 software.

\section{Results}

\subsection{Probe length is a key factor for microarray detection of single nucleotide variations in BRSV genome}

To reveal the presence of single nucleotide variants in BRSV genome populations by oligonucleotide-based microarrays, target DNAs were amplified from BRSV phosphoprotein $\mathrm{P}$ genomic region cloned into E. coli plasmids. Phosphoprotein $\mathrm{P}$ sequences were derived from two BRSV populations (W2BT10 and W2HEp58), which are characterized by a T/G polymorphism at genome position 2686 .

A first set of oligonucleotide probes ranging from 14 to 17 mers were designed with the discriminating nucleotide positioned at the centre of the probe (Table 1) and printed on epoxy-activated glass slides. This short oligonucleotide strategy was chosen in accordance with a recent report showing the detection of antigenic variants of foot-and-mouth disease virus (FMDV) using 15-mers oligoprobes with oligo (dT)15 spacer (Martin et al., 2006). Hybridization with Cy5-labelled target DNA with these short oligoprobes was not specific, and neither addition of a poly dT spacer at oligonucleotide $5^{\prime}$ extremity, nor elevation of the hybridization temperature $\left(65^{\circ} \mathrm{C}\right)$ could improve the signal intensity and the specificity of the hybridization on the parental DNA target. Therefore, these experiments were repeated using longer oligonucleotides from 19 to 23 mer spotted on Nexterion ${ }^{\circledR}$ HiSens E (Schott) slide. In these conditions, $2 \mathrm{~h}$ hybridization at $37^{\circ} \mathrm{C}$ was sufficient to obtain an intense fluorescence signal at each spot, although the absolute intensity was dependent upon the length of the oligonucleotide probes, with a maximal hybridization signal on the 23 mer probes (Fig. 2). However, discrimination between T2686 and G2686 target DNAs, as determined by the $\mathrm{T} / \mathrm{G}$ signal ratio, was optimal with the 19 mer oligoprobes (Fig. 3). The T/G signal ratio was not enhanced by using purified target DNA (PCR products), whereas target signal intensities were strongly reduced after the purification procedure, probably as a result of the loss of labelled material. As expected, no hybridization was observed between Cy5-labelled target DNA and oligoprobes designed on the F70 and F218 BRSV genomic regions, as they mapped outside of the phosphoprotein $\mathrm{P}$ coding region.
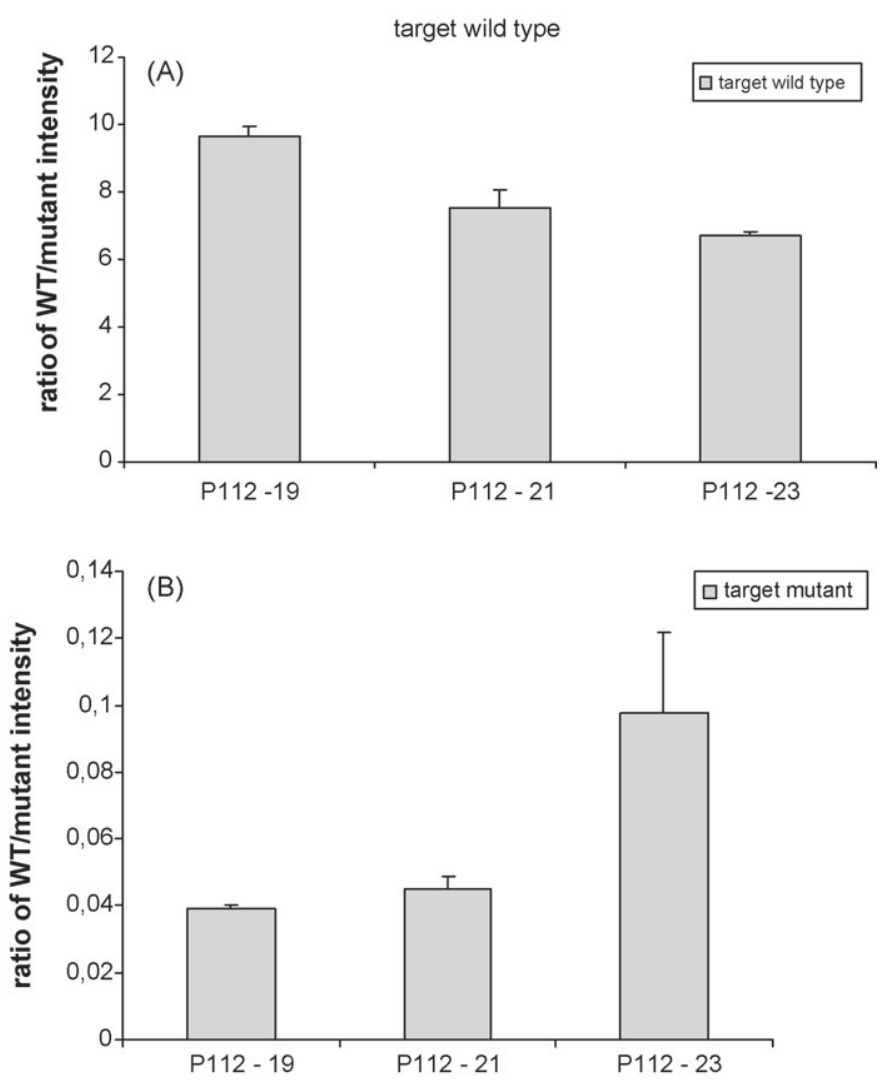

Fig. 3. Influence of the probe sizes on the hybridization specificity of BRSV parental and mutant genome. The target BRSV wild type (A) and BRSV mutant (B) were produced as in Fig. 2. They were hybridized at $37^{\circ} \mathrm{C}$ for $4 \mathrm{~h}$ and the specificity was expressed as the ratio of the BRSV parental hybridization signal vs. that of the mutant on each oligoprobe. 

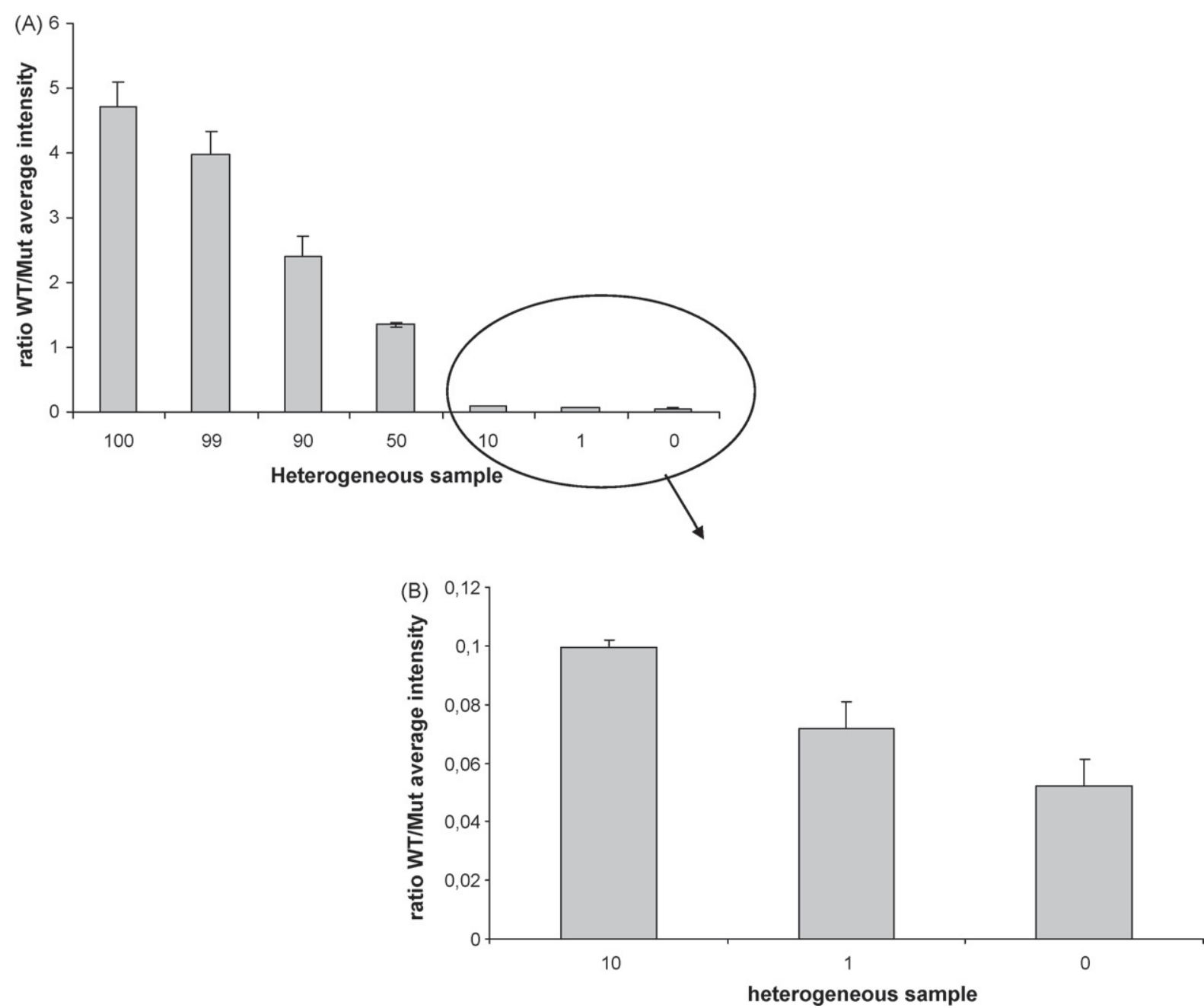

Fig. 4. Discrimination rate of BRSV parental cDNA vs. BRSV mutant cDNA. The Cy5-labelled targets from BRSV parental and mutant genome were produced by RT-PCR as in Fig. 2. The parental and mutant amplicons were mixed at various ratios as indicated in Table 2, and were hybridized on a 21 mer oligoprobes at $55^{\circ} \mathrm{C}$ for $2 \mathrm{~h}$. (B) Corresponds to a zoom of the histogram (A) for heterogeneous samples 10,1 and 0. The fluorescent signals were captured using laser scanner Axon GenePix 4000A with a PMT of 600 at $635 \mathrm{~nm}$ and 450 at $532 \mathrm{~nm}$. The discrimination was expressed as the ratio of hybridization signal between BRSV parental and mutant genome.

\subsection{Sensitivity limit for discrimination of mutant variants within BRSV populations}

Data given above showed that the parental and the mutant BRSV genome at position 2686 could be discriminated using 19 or 21 mer oligonucleotides. The discriminative sensitivity was quantified by mixing different proportions of parental and mutant cDNA before performing a labelled PCR on this prepared mix (Table 2). These PCR mixtures were subsequently hybridized to a microchip made of 21-mer oligonucleotides. As shown in Fig. 4, a proportion of as low as $1 \%$ of the parental amplicon in the total BRSV amplicon population gave a statistically significant, elevated fluorescent signal in comparison to residual fluorescent signal obtained with a population made of $100 \%$ of the mutant amplicon. This discrimination rate was around 10 times higher than the one reported by Proudnikov et al. (2000) for the population of oral poliovirus vaccine, probably because these authors used a generic microchips consisting in all possible hexamers flanked at both ends of an octanucleotide for detection of the neurovirulent mutants in oral poliovirus vaccine. The discrimination rate between two different targets DNA hybridizing the same probes was also comparable to that of a same target hybridizing two oligoprobes that differed from each other by a single point mutation (Le Berre et al., 2003).

\section{Discussion}

Recent progress in pathogen genomics and microarray technology has allowed the development of high throughput tools for the rapid and accurate detection of human, animal and plant pathogens. Volokhov et al. (2002) used oligonucleotide microarrays for the detection and discrimination of six species among the Listeria genus based on sequence differences in the iap gene. Microarrays bearing 40-70 mer oligonucleotides were also designed for the genotyping of human and plant viral pathogens (Wang et al., 2002; Bystricka et al., 2005). More recently, Palacios et al. (2007) reported the development of a 
panmicrobial microarray (GreeneChipPm) consisting of 60-mer oligonucleotides probes for diagnosis of a large collection of pathogens.

In all cases, these microarrays were designed for the detection of various microbes or species and the specificity was based on the oligoprobes design. Nevertheless, the current literature reveals few quantitative DNA microarray-based analyses of mutant genome distributions within viral populations. This question has been addressed in this present case study aiming at quantifying the heterogeneity of RNA genomes in viral populations. The strategy that used short 21 mer oligonucleotides was found to be effective, because the discrimination rate between the two genomes was around $1 \%$. This resolution was in the same order of magnitude as a classical RT-PCR and the recently developed MAPREC technique.

Taken together, these results should encourage future development for more complex oligonucleotide arrays to investigate the genetic variability within viral population in a high throughput manner, and to use them for the screening of viral isolates from various sources, including healthy carriers, animals and plants.

\section{Acknowledgments}

Dr. Andrew Mc Donagh from the Imperial College of London is acknowledged for his English proofreading of the manuscript, and Mr. Moulignie for his excellent technical assistance. This work was supported in part by the ACI 'Microbiologie 2003' of the French Ministry of Education and Research to Eric Baranowski and by the 'Reseau National des Genopoles' to Veronique Le Berre and Jean-Marie François.

\section{References}

Amexis, G., Oeth, P., Abel, K., Ivshina, A., Pelloquin, F., Cantor, C.R., Braun, A., Chumakov, K., 2001. Quantitative mutant analysis of viral quasispecies by chip-based matrix-assisted laser desorption/ionization timeof-flight mass spectrometry. Proc. Natl. Acad. Sci. U.S.A. 98 (21), $12097-$ 12102.

Bystricka, D., Lenz, O., Mraz, I., Piherova, L., Kmoch, S., Sip, M., 2005 Oligonucleotide-based microarray: a new improvement in microarray detection of plant viruses. J. Virol. Methods 128 (1-2), 176-182.
Chumakov, K.M., Norwood, L.P., Parker, M.L., Dragunsky, E.M., Ran, Y.X., Levenbook, I.S., 1992. RNA sequence variants in live poliovirus vaccine and their relation to neurovirulence. J. Virol. 66, 966-970.

Deplanche, M., Lemaire, M., Mirandette, C., Bonnet, M., Schelcher, F., Meyer, G., 2007. In vivo evidence for quasispecies distributions in the bovine respiratory syncytial virus genome. J. Gen. Virol. 88, 1260-1265.

Domingo, E., Martin, V., Perales, C., Grande-Pérez, A., García-Arriaza, J., Arias, A., 2006. Viruses as quasispecies: biological implications. Curr. Top. Microbiol. Immunol. 299, 51-82.

Domingo, E., Gomez, J., 2007. Quasispecies and its impact on viral hepatitis. Virus Res. 127, 131-150.

Le Berre, V., Trevisiol, E., Dagkessamanskaia, A., Sokol, S., Caminade, A.M. Majoral, J.P., Meunier, B., François, J., 2003. Dendrimeric coating of glass slides for sensitive DNA microarrays analysis. Nucleic Acids Res. 31 (16), $1-8$.

Martin, V., Perales, C., Abia, D., Ortiz, A.R., Domingo, E., Briones, C., 2006. Microarray-based identification of antigenic variants of foot-and-mouth disease virus: a bioinformatics quality assessment. BMC Genom. 7, 117.

Palacios, G., Quan, P.L., Jabado, O.J., Conlan, S., Hirschberg, D.L., Liu, Y., Zhai, J., Renwick, N., Hui, J., Hegyi, H., Grolla, A., Strong, J.E., Towner, J.S., Geisbert, T.W., Jahrling, P.B., Buchen-Osmond, C., Ellerbrok, H., SanchezSeco, M.P., Lussier, Y., Formenty, P., Nichol, M.S., Feldmann, H., Briese, T., Lipkin, W.I., 2007. Panmicrobial oligonucleotide array for diagnosis of infectious diseases. Emerg. Infect. Dis. 13 (1), 73-81.

Proudnikov, D., Kirillov, E., Chumakov, K., Donlon, J., Rezapkin, G., Mirzabekov, A., 2000. Analysis of mutations in oral poliovirus vaccine by hybridization with generic oligonucleotide microchips. Biologicals 28 (2), 57-66.

Ryabinin, V.A., Shundrin, L.A., Kostina, L.B., Laassri, M., Chizhikov, V., Shchelkunov, S.N., Chumakov, C., Sinyakov, A.N., 2006. Microarray assay for detection and discrimination of Orthopoxvirus species. J. Med. Virol. 78 (10), 1325-1340.

Valarcher, J.F., Bourhy, H., Gelfi, J., Schelcher, F., 1999. Evaluation of a nested reverse transcription-PCR assay based on the nucleoprotein gene for diagnosis of spontaneous and experimental bovine respiratory syncytial virus infections. J. Clin. Microbiol. 37 (6), 1858-1862.

Volokhov, D., Rasooly, A., Chumakov, K., Chizhikov, V., 2002. Identification of Listeria species by microarray-based assay. J. Clin. Microbiol. 40 (12), 4720-4728.

Wang, D., Coscoy, L., Zylberberg, M., Avila, P.C., Boushey, H.A., Ganem, D., DeRisi, J.L., 2002. Microarray-based detection and genotyping of viral pathogens. Proc. Natl. Acad. Sci. 99, 15687-15692.

Warsen, A.E., Krug, M.J., LaFrentz, S., Stanek, D.R., Loge, F.J., Call, D.R., 2004. Simultaneous discrimination between 15 fish pathogens by using $16 \mathrm{~S}$ ribosomal DNA PCR and DNA microarrays. Appl. Environ. Microbiol. 70, 4216-4221.

Wood, D.J., Macadam, A.J., 1997. Laboratory tests for live attenuated poliovirus vaccines. Biologicals 25, 3-15. 